\title{
ERRATUM
}

Mikiko Nakagawa $\cdot$ Nario Sano $\cdot$ Seiki Nobe

Masashi Ichinose · Hidetoshi Yonemochi

Tetsunori Saikawa

\section{Patients with mild hypertensive heart disease and left ventricular outflow tract obstruction: treatment with angiotensin II antagonists}

\section{Heart Vessels (2004) 19:248-251}

The article cited above should have appeared as a Case report, not as an Original article.

The publisher sincerely apologizes for the error. 